BULLETIN OF THE POLISH

ACADEMY OF SCIENCES

MATHEMATICS

Vol. 67, No. 2, 2019

\title{
On the existence of almost disjoint and MAD families without AC
}

by

\section{Eleftherios TACHTSIS}

Presented by Feliks PRZYTYCKI

Summary. In set theory without the Axiom of Choice (AC), we investigate the deductive strength and mutual relationships of the following statements:

(1) Every infinite set $X$ has an almost disjoint family $\mathcal{A}$ of infinite subsets of $X$ with $|\mathcal{A}| \not \leq \aleph_{0}$.

(2) Every infinite set $X$ has an almost disjoint family $\mathcal{A}$ of infinite subsets of $X$ with $|\mathcal{A}|>\aleph_{0}$.

(3) For every infinite set $X$, every almost disjoint family in $X$ can be extended to a maximal almost disjoint family in $X$.

(4) For every infinite set $X$, no infinite maximal almost disjoint family in $X$ has cardinality $\aleph_{0}$.

(5) For every infinite set $A$, there is a continuum sized almost disjoint family $\mathcal{A} \subseteq A^{\omega}$.

(6) For every free ultrafilter $\mathcal{U}$ on $\omega$ and every infinite set $A$, the ultrapower $A^{\omega} / \mathcal{U}$ has cardinality at least $2^{\aleph_{0}}$.

1. Introduction. It is part of the folklore that $\omega$ (the set of natural numbers) has a continuum sized (and thus uncountable) almost disjoint family (complete definitions will be given in Section 2); no maximal almost disjoint (MAD) family in $\omega$ is countable; and any almost disjoint family in $\omega$ can be extended to a MAD family in $\omega$. The first two of the above results are provable in ZF, i.e. in Zermelo-Fraenkel set theory without AC, while the third one follows easily from Zorn's Lemma (which is equivalent to AC).

2010 Mathematics Subject Classification: 03E05, 03E25, 03E35.

Key words and phrases: Axiom of Choice, weak axioms of choice, almost disjoint family, MAD family, models of ZFA and ZF, Pincus' transfer theorems, Jech-Sochor First Embedding Theorem.

Received 16 February 2018; revised 29 March 2019.

Published online 30 May 2019. 
Therefore, an immediate question that arises is whether AC is essential for the proof of the latter result. It is a notable recent result (solving a longstanding problem by Mathias [8]) due to Törnquist [11] that in Solovay's model (Model $\mathcal{M} 5(\aleph)$ in Howard and Rubin [5]) there are no infinite MAD families in $\omega$ (recall also that Solovay's model is constructed by starting with a ground model with an inaccessible cardinal), and thus the extension of almost disjoint families in $\omega$ to MAD families cannot be derived from the ZF axioms alone. Further remarkable progress was made by Horowitz and Shelah [4] who eliminated the large cardinal assumption from Törnquist's result by establishing that ZF + DC + "There are no MAD families in $\omega$ " is equiconsistent with ZFC (where ZFC is ZF + AC and DC is the Principle of Dependent Choices).

While the above focuses on the definability of MAD families in $\omega$ in certain models of $\mathrm{ZF}+\neg \mathrm{AC}$, the research in this paper - motivated by the above three results on $\omega$-centers around the natural, and open so far, questions which naturally arise on the set-theoretic strength of relative statements about almost disjoint and MAD families in any infinite set in mild extensions of ZF and ZFA (ZF with the Axiom of Extensionality modified in order to allow atoms), that is, in ZF + Weak Choice and in ZFA + Weak Choice.

From this perspective, the current project fills an important gap in information by providing a plethora of results which shed light on the problem of the placement of statements such as: "Every infinite set has an uncountable almost disjoint family"; "Every almost disjoint family in an infinite set $X$ can be extended to a MAD family in $X$ "; "No infinite MAD family in an infinite set has cardinality $\aleph_{0}$ "; and "For every every infinite set $X$, there is a continuum sized almost disjoint family $\mathcal{X} \subseteq X^{\omega}$ " in the hierarchy of weak choice principles, as well as of their mutual relationship.

As is probably expected (and taking into account the results by Törnquist, Horowitz and Shelah), none of those statements are provable in ZF. This is indeed the case and our main purpose is to provide as much information as possible about implications/non-implications that hold true between the above principles and several consequences of AC. Besides the positive results of this paper which depict the set-theoretic assumptions that are sufficient for the proofs of the statements of discourse, there is a considerable amount of central results which are ZFA independence results (see Theorems 2(ii, iii); 3(iii, iv, v); 4 5 (iii, iv); 6). The majority of these results can be transferred to ZF using the transfer theorems of Pincus [9] or the Jech-Sochor First Embedding Theorem (see [6, Theorem 6.1]). For the independence in ZFA, we employ renowned Fraenkel-Mostowski (FM) permutation models and their techniques; two exceptions are the FM models of the proof of Theorem 5(iii) and of Remark 2, which are introduced in this paper. Whenever the transfer to ZF is possible, we will mention in the statement of the theo- 
rem that the independence is in ZF and we will provide within the relevant proof a brief outline of the transfer as well as references to the literature. For a detailed account of permutation models, the reader is referred to Jech's classical book [6, Chapter 4].

For the reader's convenience, we highlight most of the core results of this paper in the list below.

(1) "Every infinite set has an uncountable almost disjoint family" is deducible from each of "Every Dedekind-finite set is finite" (DF $=F$ ) and the Axiom of Multiple Choice (MC), but it is not deducible from the Boolean Prime Ideal Theorem (BPI) or "For every infinite set $X, \mathcal{P}(X)$ is Dedekindinfinite" (Theorems 2, 3).

(2) MC implies "For every infinite set $X$, every almost disjoint family in $X$ can be extended to a MAD family in $X$ ", but the implication is not reversible in ZFA (Theorems 5(i) and 6).

(3) "The power set of a well-ordered set can be well-ordered" does not imply "For every infinite set $X$, every almost disjoint family in $X$ can be extended to a MAD family in $X$ " in ZFA (Theorem 5 (iii)).

(4) The Axiom of Countable Multiple Choice $\left(M C^{\aleph_{0}}\right)$ is equivalent to "For every infinite set $X$, no infinite MAD family in $X$ has cardinality $\aleph_{0}$ " (Theorem 7).

(5) $\mathrm{DF}=\mathrm{F}$ is equivalent to "For every every infinite set $A$, there is a continuum sized almost disjoint family $\mathcal{A} \subseteq A^{\omega}$ ", which in turn strictly implies "For every free ultrafilter $\mathcal{U}$ on $\omega$ and every infinite set $A,\left|A^{\omega} / \mathcal{U}\right| \geq 2^{\aleph_{0}}$ " (Theorem 8, Corollary 1, and Theorem 10(iii)).

2. Notation, terminology, and known results. We start with the definitions of almost disjoint family and maximal almost disjoint family of infinite subsets of a given infinite set $X$. Our definition of almost disjoint family differs from the usual one which states that a family $\mathcal{A} \subseteq[X]^{|X|}=$ $\{Y \subseteq X:|Y|=|X|\}$ is almost disjoint in $X$ if for any distinct $A, B \in \mathcal{A}$, $|A \cap B|<|X|$ (that is, there is a one-to-one mapping from $A \cap B$ into $X$ but no one-to-one mapping from $X$ into $A \cap B$ ); we shall only be interested in the case where all elements of $\mathcal{A}$ are infinite sets and $A \cap B$ is finite for any distinct $A, B \in \mathcal{A}$.

Definition 1. Let $X$ be an infinite set. (That is, $X \neq \emptyset$ and for all $n \in \omega \backslash\{0\}$, there is no bijection $f: n \rightarrow X$; otherwise $X$ is called finite.)

A family $\mathcal{A}$ of infinite subsets of $X$ is called almost disjoint in $X$ if for all $A, B \in \mathcal{A}$ with $A \neq B$, the set $A \cap B$ is finite. An almost disjoint family $\mathcal{A}$ in $X$ is called maximal almost disjoint (MAD) in $X$ if for every almost disjoint family $\mathcal{B}$ in $X$ with $\mathcal{A} \subseteq \mathcal{B}$, we have $\mathcal{A}=\mathcal{B}$. 
Definition 2. Let $A$ and $X$ be non-empty sets and also let $\mathcal{F}$ be a filter on $X$. For $f, g \in A^{X}$ (the set of all functions from $X$ into $A$ ) define

$$
f \sim_{\mathcal{F}} g \Longleftrightarrow\{x \in X: f(x)=g(x)\} \in \mathcal{F} .
$$

Since $X \in \mathcal{F}$ and $\mathcal{F}$ is closed with respect to finite intersections, it is easy to see that $\sim_{\mathcal{F}}$ is an equivalence relation on $A^{X}$. Let

$$
[f]=\left\{g \in A^{X}: f \sim_{\mathcal{F}} g\right\}
$$

(i.e. $[f]$ is the equivalence class of $f$ under $\sim_{\mathcal{F}}$ ) and let

$$
A^{X} / \mathcal{F}=\left\{[f]: f \in A^{X}\right\} .
$$

Definition 3. A set $X$ is called Dedekind-finite if $\aleph_{0} \not \leq|X|$, i.e. there is no one-to-one function $f: \omega \rightarrow X$. Otherwise, $X$ is called Dedekind-infinite.

An infinite set $X$ is called amorphous if $X$ cannot be written as a disjoint union of two infinite subsets. Clearly, every amorphous set is Dedekind-finite, but the converse may fail; the set $A$ of the countably many added Cohen reals in the Basic Cohen Model, is Dedekind-finite but not amorphous - see [5. Model $\mathcal{M} 1$.

A set $X$ is called uncountable if $|X| \not \leq \aleph_{0}$ (that is, $X$ is uncountable if there is no one-to-one function $f: X \rightarrow \omega$ ).

Another possible definition of uncountable is the following: A set $X$ is uncountable if $\aleph_{0}<|X|$, i.e. if there is a one-to-one function $f: \omega \rightarrow X$, but there is no one-to-one function $g: X \rightarrow \omega$. It is clear that the latter definition implies the former (recall here that the Cantor-Bernstein Theorem, that if $|A| \leq|B|$ and $|B| \leq|A|$ then $|A|=|B|$, is provable in ZF), and that $(\forall X)\left(|X| \not \leq \aleph_{0} \Leftrightarrow \aleph_{0}<|X|\right)$ is equivalent to "Every Dedekind-finite set is finite".

In what follows, whenever we use the term 'uncountable' we will assume the meaning of the first definition (i.e. $|X| \not \leq \aleph_{0}$ ), and whenever we use the second definition (i.e. $\aleph_{0}<|X|$ ), we will write 'uncountable $\left(>\aleph_{0}\right)$ '.

Next, we list the choice principles to be used in this paper.

DeFinition 4.

1. The Axiom of Choice AC (Form 1 in [5]): Every set of non-empty sets has a choice function.

2. The Axiom of Multiple Choice MC (Form 67 in [5]): For every set $X$ of non-empty sets there is a function $F$ with domain $X$ such that for all $x \in X, f(x)$ is a non-empty finite subset of $x$. The function $F$ is called a multiple choice function of $X$.

$\mathrm{MC}$ is equivalent to $\mathrm{AC}$ in $\mathrm{ZF}$, but it is not equivalent to $\mathrm{AC}$ in ZFA (see [5], [6, Theorems 9.1 and 9.2]). 
3. The Axiom of Countable Multiple Choice $\mathrm{MC}^{\aleph_{0}}$ (Form 126 in [5]): Every denumerable (= countably infinite) family of non-empty sets has a multiple choice function.

$M C^{\aleph_{0}}$ is equivalent to its partial version $\mathrm{PMC}^{\aleph_{0}}$ : Every denumerable family $\mathcal{A}$ of non-empty sets has a partial multiple choice function, that is, $\mathcal{A}$ has an infinite subfamily with a multiple choice function (see [5]).

4. The Axiom of Countable Choice $\mathrm{AC}^{\aleph_{0}}$ (Form 8 in [5]): Every denumerable family of non-empty sets has a choice function.

$A C^{\aleph_{0}}$ is equivalent to its partial version $\operatorname{PAC}^{\aleph_{0}}$ (see [5]).

5. $A C_{\text {fin }}^{\aleph_{0}}($ Form 10 in [5]): Every denumerable set of non-empty finite sets has a choice function.

$A C_{\text {fin }}^{\aleph_{0}}$ is equivalent to its partial version $\operatorname{PAC}_{\text {fin }}^{\aleph_{0}}$ (see [5]).

6. PW (Form 91 in [5]): The power set of a well-ordered set can be wellordered.

$\mathrm{PW}$ is equivalent to $\mathrm{AC}$ in $\mathrm{ZF}$, but it is not equivalent to $\mathrm{AC}$ in ZFA (see [5], [6, Theorems 9.1 and 9.2]).

7. The Boolean Prime Ideal Theorem BPI (Form 14 in [5]): Every Boolean algebra has a prime ideal. Equivalently, every proper filter on a set $X$ can be extended to an ultrafilter on $X$ (Form [14 A] in [5]).

8. $\operatorname{UF}(\omega)$ (Form 70 in [5]): There is a free ultrafilter on $\omega$.

9. $\mathrm{DF}=\mathrm{F}($ Form 9 in [5]): Every Dedekind-finite set is finite.

10. $\mathrm{LDF}=\mathrm{F}($ Form 185 in [5]): Every linearly ordered Dedekind-finite set is finite.

11. $\mathrm{AC}_{\mathrm{DLO}}^{\aleph_{0}}$ : Every denumerable family of defined linear orders has a choice function (i.e. if $\mathcal{A}=\left\{\left(A_{i}, \leq_{i}\right): i \in \omega\right\}$ is a denumerable family of linearly ordered sets, then there is a choice function of $\left\{A_{i}: i \in \omega\right\}$ ). (The weak choice principle $A C_{D L O}^{\aleph_{0}}$ is introduced here.)

The subsequent theorem is part of the folklore.

THEOREM 1. The following hold:

(i) (ZF) There is a continuum sized almost disjoint family in $\omega$.

(ii) (ZFC) For every infinite set $X$, every almost disjoint family in $X$ can be extended to a MAD family.

Next, we recall a characterization of MC by A. Lévy [7].

LEMMA 1 (Lévy's Lemma). MC if and only if every infinite set can be written as a well-orderable disjoint union of non-empty finite sets. 
3. Main results. The following theorem provides information on the deductive strength of the statement "Every infinite set has an uncountable almost disjoint family". As will be clarified, this statement cannot be proved without using some form of choice.

TheOREM 2. The following hold:

(i) $\mathrm{DF}=\mathrm{F}$ implies "Every infinite set has an uncountable almost disjoint family", which in turn implies "There are no amorphous sets". None of the above implications are reversible in ZF.

(ii) In ZFA, MC implies "Every infinite set has an uncountable almost disjoint family". Hence, the latter statement does not imply $\mathrm{AC}_{\text {fin }}^{\aleph_{0}}$ (and hence $\mathrm{DF}=\mathrm{F})$ in $\mathrm{ZF}$, and does not imply $\mathrm{MC}$ in $\mathrm{ZF}$ either.

(iii) $\mathrm{BPI}$ does not imply "Every infinite set has an uncountable almost disjoint family" in ZF.

Proof. (i) Assume that DF $=\mathrm{F}$ is true. Let $X$ be an infinite set. By our assumption, $X$ has a countably infinite subset, say $Y=\left\{y_{n}: n \in \omega\right\}$. By Theorem 1, it follows that $Y$ has an uncountable almost disjoint family. Hence, so does its superset $X$.

The second implication of (i) follows immediately from the facts that the only subsets of an amorphous set are the finite and the co-finite ones and that any two distinct members $A$ and $B$ of an almost disjoint family have the property that $A \backslash B$ and $B \backslash A$ are both infinite.

For "There are no amorphous sets" $\nrightarrow$ "Every infinite set has an uncountable almost disjoint family of infinite subsets" in ZF, see the forthcoming proof of part (iii) (and note that BPI implies there are no amorphous sets).

For "Every infinite set has an uncountable almost disjoint family of infinite subsets" $\nrightarrow \mathrm{DF}=\mathrm{F}$ in $\mathrm{ZF}$, see the proof of part (ii) below.

(ii) Assume that $\mathrm{MC}$ is true. Let $X$ be an infinite set. By (Lévy's) Lemma 1, $X$ has a well-ordered partition $\mathcal{U}=\left\{X_{\alpha}: \alpha<\kappa\right\}(\kappa$ an infinite well-ordered cardinal number) into non-empty finite sets. By Theorem 1 , there exists a continuum sized almost disjoint family of $\mathcal{V}:=\left\{X_{n}: n \in \omega\right\}$, say $\mathcal{A}$. Then $\mathcal{Z}=\{\bigcup A: A \in \mathcal{A}\}$ is clearly a continuum sized (and thus uncountable) family of infinite subsets of $X$ which is almost disjoint.

For the last assertion of (ii), we first note that in the Second Fraenkel Model (Model $\mathcal{N} 2$ in [5]), $M C$ is true but there is a countably infinite family of pairwise disjoint sets of pairs of atoms which has no partial choice function in $\mathcal{N} 2$ (see [5], [6]). Hence, $\Psi=$ "Every infinite set has an uncountable almost disjoint family of infinite subsets" is true in $\mathcal{N} 2$, whereas $A C_{\text {fin }}^{\aleph_{0}}$ is false. Furthermore, $\Psi$ is injectively boundable since by part (i) of the current theorem, it follows that

$\Psi \Longleftrightarrow(\forall x)\left(|x|_{-} \leq \omega \rightarrow x\right.$ has an uncountable almost disjoint family) 
(for the terms 'injectively boundable' and 'injective cardinality $|x|+$ ', see [5, Note 103] or Pincus [9]). Since $\Phi=\Psi \wedge \neg A C_{\text {fin }}^{\aleph_{0}}$ is a conjunction of injectively boundable statements and has a ZFA model, it follows from Theorem 4 of Pincus [9] that $\Phi$ has a ZF model.

The fact that $\Psi$ does not imply $M C$ in ZF follows from (i) and the fact that $\mathrm{DF}=\mathrm{F}$ is strictly weaker than $\mathrm{AC}$ in $\mathrm{ZF}$.

(iii) We first prove independence in ZFA. Then the result can be transferred to ZF using Pincus' transfer theorems. To this end, we will show that in Mostowski's Linearly Ordered Model (Model $\mathcal{N} 3$ in [5]), which satisfies BPI (see [5]), there exists an infinite set which has no infinite almost disjoint families. Let us recall the description of $\mathcal{N} 3$ : We start with a model $M$ of ZFA + AC with a countable set $A$ of atoms using an ordering $\leq$ of $A$ chosen so that $(A, \leq)$ is order isomorphic to the set $\mathbb{Q}$ of rational numbers with the usual ordering. Let $G$ be the group of all order automorphisms of $(A, \leq)$. For any element $x$ of $M, \operatorname{fix}_{G}(x)$ denotes the (pointwise stabilizer) subgroup $\{\phi \in G:(\forall t \in x)(\phi(t)=t)\}$ of $G$ and $\operatorname{Sym}_{G}(x)$ denotes the (stabilizer) subgroup $\{\phi \in G: \phi(x)=x\}$ of $G$. Let $\Gamma$ be the finite support normal filter, i.e. $\Gamma$ is the filter of subgroups of $G$ generated by the subgroups $\left\{\operatorname{fix}_{G}(E): E \in[A]^{<\omega}\right\}$, where $[A]^{<\omega}$ is the set of finite subsets of $A$. An element $x$ of $M$ is called symmetric if $\operatorname{Sym}_{G}(x) \in \Gamma$; hence $x$ is symmetric if there is some finite set $E \subset A$ such that $\operatorname{fix}_{G}(E) \subseteq \operatorname{Sym}_{G}(x)$. The set $E$ is then called a support of $x$. The element $x$ of $M$ is called hereditarily symmetric if $x$ and all elements in its transitive closure are symmetric. Then $\mathcal{N} 3$ is the Fraenkel-Mostowski model determined by $M, G$ and $\Gamma$, that is, $\mathcal{N} 3$ consists exactly of all the hereditarily symmetric elements of $M$.

We will prove that $A$ has no infinite almost disjoint family in $\mathcal{N} 3$. Assume the contrary, so let $\mathcal{A}$ be an infinite almost disjoint family of infinite subsets of $A$. Let $E=\left\{e_{1}, \ldots, e_{k}\right\} \subset A$, where $e_{1}<\cdots<e_{k}$, be a finite support of $\mathcal{A}$. Consider the following intervals in the ordering $\leq$ of $A: I_{0}=\left(-\infty, e_{1}\right)$, $I_{i}=\left(e_{i}, e_{i+1}\right)$ where $i=1, \ldots, k-1, I_{k}=\left(e_{k+1},+\infty\right)$. Since $\mathcal{A}$ is infinite and every member of $\mathcal{A}$ is infinite, it follows that there exists an element $B \in \mathcal{A}$ such that for some $i<k+1, B \cap I_{i}$ is infinite and $I_{i} \backslash B$ is infinite. It is fairly straightforward now to construct an order automorphism $\phi$ of $A$ such that $\phi \in \operatorname{fix}_{G}(E), \phi(B) \neq B$ and $\phi(B) \cap B$ is infinite. Since $E$ is a support of $\mathcal{A}$, it follows that $\phi(\mathcal{A})=\mathcal{A}$ and hence $\phi(B) \in \mathcal{A}$. Since $\phi(B) \cap B$ is infinite, we obtain a contradiction to the fact that $\mathcal{A}$ is almost disjoint. Therefore, in $\mathcal{N} 3$, there is no infinite almost disjoint family in $A$, finishing the proof.

Now, since the statement $\Psi=$ "There exists an infinite set which has no infinite almost disjoint family" is injectively boundable (for it is boundable), and $\Phi=\operatorname{BPI} \wedge \Psi$ is a conjunction of BPI and an injectively boundable 
statement which has a ZFA model, it follows from Theorem 4 of Pincus [9] that $\Phi$ also has a ZF model (see also [5, Note 103, p. 286]). This completes the proof of (iii) and of the theorem.

From Theorem 2 and its proof, we immediately obtain most of the results of the following theorem.

TheOrem 3. The following hold:

(i) Assume that DF = F is true. Then "Every infinite set has an uncountable almost disjoint family" is equivalent to "Every infinite set has an uncountable $\left(>\aleph_{0}\right)$ almost disjoint family".

(ii) $\mathrm{DF}=\mathrm{F}$ implies "Every infinite set has an uncountable $\left(>\aleph_{0}\right)$ almost disjoint family", which in turn implies "For every infinite set $X, \mathcal{P}(X)$ is Dedekind-infinite", which implies "There are no amorphous sets".

(iii) "For every infinite set $X, \mathcal{P}(X)$ is Dedekind-infinite" does not imply "Every infinite set has an uncountable almost disjoint family" in ZF, and thus it does not imply "Every infinite set has an uncountable $\left(>\aleph_{0}\right)$ almost disjoint family" in ZF either.

(iv) In ZFA, MC implies "Every infinite set has an uncountable $\left(>\aleph_{0}\right)$ almost disjoint family". Hence, the latter statement does not imply $\mathrm{AC}_{\mathrm{fin}}^{\aleph_{0}}$ (and hence $\mathrm{DF}=\mathrm{F}$ ) in $\mathrm{ZF}$, and does not imply $\mathrm{MC}$ in $\mathrm{ZF}$ either.

(v) BPI does not imply "Every infinite set has an uncountable $\left(>\aleph_{0}\right)$ almost disjoint family" in ZF.

Proof. We shall only discuss the proofs of (i) and (iii) (see the paragraph prior to the current theorem).

(i) This follows from the fact that under the assumption of DF $=F$, uncountable is equivalent to uncountable $\left(>\aleph_{0}\right)$.

(iii) We first show independence in ZFA. The permutation model to be used is the Höft/Howard Model $\mathcal{N} 46$ in [5], whose description is as follows: We begin with a model $M$ of ZFA + AC with a countable set $A$ of atoms and an ordering $\leq$ of $A$ such that $(A, \leq)$ has the same order type as that of the rational numbers. We assume that $A$ is the disjoint union of three dense subsets $D_{1}, D_{2}$ and $D_{3}$. We let $G$ be the group of all order automorphisms $\phi$ of $(A, \leq)$ such that $\phi\left(D_{i}\right)=D_{i}, i=1,2,3$. A subset $E$ of $A$ will be called a support if it satisfies the following conditions:

1. $E \cap D_{1}$ is finite.

2. $E \cap D_{2}$ is well-ordered by $\leq$.

3. If $b: \alpha \rightarrow E \cap D_{2}$ is an order preserving bijection from an ordinal $\alpha$ onto $E \cap D_{2}$ and $\lambda \leq \alpha$ is a limit ordinal, then the least upper bound of $\{b(\gamma): \gamma<\lambda\}$ in $(A, \leq)$ exists and is in $D_{3}$.

4. $E \cap D_{3}=\emptyset$. 
$\Gamma$ is the normal filter of subgroups of $G$, which is generated by the filter base $\left\{\operatorname{fix}_{G}(E): E \subset A\right.$ is a support $\}$. Then $\mathcal{N} 46$ is the Fraenkel-Mostowski model of ZFA which is determined by $M, G$ and $\Gamma$.

In [3, Theorem 3.6], Höft and Howard proved that if $X$ is an infinite set in $\mathcal{N} 46$, then $\mathcal{P}(X)$ is Dedekind-infinite.

Now almost identically to the proof that in Mostowski's Linearly Ordered Model $\mathcal{N} 3$, the set $A$ of atoms has no infinite almost disjoint families, it can be shown (by taking into account condition 1 satisfied by a support) that in $\mathcal{N} 46$, the subset $D_{1}$ has no infinite almost disjoint families in $\mathcal{N} 46$.

To end the proof, we note that "For every infinite set $X, \mathcal{P}(X)$ is Dedekind-infinite" is injectively boundable for it is equivalent to

$$
\text { " }(\forall x)\left(|x|_{-} \leq \omega \rightarrow \mathcal{P}(x) \text { is Dedekind-infinite }\right) " .
$$

Since $\Phi=$ "For every infinite set $X, \mathcal{P}(X)$ is Dedekind-infinite" $\wedge$ "There exists an infinite set which has no infinite almost disjoint family" is a conjunction of injectively boundable statements, which has a ZFA model, it follows from Pincus' transfer theorems that $\Phi$ has a ZF model. This completes the proof of (iii) and of the theorem.

REMARK 1. One can think that the statement "Every infinite set has an uncountable $\left(>\aleph_{0}\right)$ almost disjoint family" is related to the principle LDF $=\mathrm{F}$, i.e. "Every linearly ordered Dedekind-finite set is finite" (note that the latter follows from each of $\mathrm{MC}$ and $\mathrm{DF}=\mathrm{F}$ ). The reason is the following: Assume that every infinite set has an uncountable $\left(>\aleph_{0}\right)$ almost disjoint family, and also there exists an infinite linearly ordered Dedekind-finite set, say $(A, \leq)$. By our assumption, there is an uncountable $\left(>\aleph_{0}\right)$ almost disjoint family $\mathcal{F}$ in $A$. Let $\mathcal{F}^{*}=\left\{F_{n}: n \in \omega\right\}$ be a denumerable subset of $\mathcal{F}$. There are two possibilities:

(i) There exists an infinite subset $\mathcal{D}$ of $\mathcal{F}^{*}$ which forms a $\Delta$-system, that is, there exists a set $r$ such that for any distinct $Y, Z \in \mathcal{D}$ we have $Y \cap Z=r$ $(r$ is called the root of the $\Delta$-system $\mathcal{D})$. Note that $r$ is finite since $\mathcal{F}$ is almost disjoint. Then $\mathcal{E}=\{D \backslash r: D \in \mathcal{D}\}$ is a disjoint denumerable family of infinite sets each of which is equipped with the linear order $\leq$ inherited by $A$. Now, if our assumption implies $\mathrm{AC}_{\mathrm{DLO}}^{\aleph_{0}}$, then any choice set of $\mathcal{E}$ is a denumerable subset of the linearly ordered set $A$, contrary to $A$ 's being Dedekind-finite.

(ii) There does not exist an infinite subset of $\mathcal{F}^{*}$ which forms a $\Delta$-system. In this case, we consider the family $\mathcal{G}=\left\{F_{n} \cap F_{m}: n<m<\omega\right\}$ and for $(n, m) \in \omega \times \omega$ with $n<m$, we let $c_{(n, m)}$ be the least element of (the finite set) $F_{n} \cap F_{m}$. Clearly, $C=\left\{c_{(n, m)}: n<m<\omega\right\}$ is a denumerable subset of $A$, contrary again to $A$ 's being Dedekind-finite.

However, we note that if $A C_{D L O}^{\aleph_{0}}$ is assumed in order to prove the above result, then the additional assumption of the statement "Every infinite set has 
an uncountable $\left(>\aleph_{0}\right)$ almost disjoint family" is redundant, that is, $\mathrm{AC}_{\mathrm{DLO}}^{\aleph_{0}}$ $\rightarrow \mathrm{LDF}=\mathrm{F}$. Indeed, let $(A, \leq)$ be an infinite linearly ordered set, and for each $n \in \omega$, let $S_{n}=\left\{f \in A^{n}: f\right.$ is one-to-one $\}$. Since $\leq$ is a linear order on $A$, each $S_{n}$ has a definable linear ordering, for example take the lexicographic order on $S_{n}$, for all $n \in \omega$. Then (by $\mathrm{AC}_{\mathrm{DLO}}^{\aleph_{0}}$ ) any choice function in $\mathcal{S}=\left\{S_{n}: n \in \omega\right\}$ easily yields a denumerable subset of $A$.

In view of the above discussion, it is reasonable to inquire whether "Every infinite set has an uncountable $\left(>\aleph_{0}\right)$ almost disjoint family" implies LDF $=\mathrm{F}$ or whether it implies $A C_{\text {DLO }}^{\aleph_{0}}$ in ZF. The answer to the first question eludes us, but we are able to answer the second one; in particular, we show next that "Every infinite set has an uncountable $\left(>\aleph_{0}\right)$ almost disjoint family" does not imply $\mathrm{AC}_{\mathrm{DLO}}^{\aleph_{0}}$ in ZF.

TheOrem 4. DF $=\mathrm{F}$ does not imply $\mathrm{AC}_{\mathrm{DLO}}^{\aleph_{0}}$ in ZF. Thus, by Theorem 3 , "Every infinite set has an uncountable $\left(>\aleph_{0}\right)$ almost disjoint family" does not imply $\mathrm{AC}_{\mathrm{DLO}}^{\aleph_{0}}$ in ZF either.

Proof. We first establish the independence result in ZFA, and the Fraenkel-Mostowski model that we will use is $\mathcal{N} 41$ of [5]. The description of the model is as follows: We start with a ground model $M$ of ZFA + AC with a set $A$ of atoms which is a denumerable disjoint union $A=\bigcup\left\{A_{n}: n \in \omega\right\}$, where each $A_{n}$ is denumerable and ordered like the rationals by $\leq_{n}$. Thus, for each $n \in \omega,\left(B_{n}, \leq_{n}\right) \simeq(\mathbb{Q}, \leq)$. Let $G$ be the group of all permutations of $A$ such that for each $n \in \omega$ and for each $\phi \in G, \phi\left\lceil A_{n}\right.$ is an order automorphism of $\left(A_{n}, \leq_{n}\right)$. Let $\Gamma$ be the normal filter of subgroups of $G$ which is generated by the pointwise stabilizers $\operatorname{fix}_{G}(E)$, where $E$ is a finite union of $A_{n}$ 's. Then $\mathcal{N} 41$ is the permutation model which is determined by $M, G$ and $\Gamma$.

Note that for each $n \in \omega, \leq \in \mathcal{N} 41$ since any permutation of $A$ in $G$ fixes $\left(A_{n}\right.$ and $) \leq_{n}$. Furthermore, $\mathcal{A}=\left\{\left(A_{n}, \leq_{n}\right): n \in \omega\right\}$ is a denumerable family of defined linear orders in $\mathcal{N} 41$, and using standard techniques in permutation models, one may easily verify that $\mathcal{A}$ has no multiple choice function in $\mathcal{N} 41$. Thus, $A C_{\mathrm{DLO}}^{\aleph_{0}}$ is false in $\mathcal{N} 41$.

Now in [5, Note 112], it is shown that DF $=F$ is true in $\mathcal{N} 41$. For the reader's convenience and to make our paper self-contained, we present an argument which is fairly similar to the one given in [5], but instead uses the following result of Truss (see [12, Theorem 3.5], and also Remark 2 below):

Lemma 2. Let $A(\mathbb{Q})$ be the group of all order automorphisms of $(\mathbb{Q}, \leq)$. If $H$ is a subgroup of $A(\mathbb{Q})$ of index less than $2^{\aleph_{0}}$, then for some finite $A \subset \mathbb{Q}$, $\operatorname{fix}(A)=H$ (i.e. $H=\{\phi \in A(\mathbb{Q}):(\forall a \in A)(\phi(a)=a)\})$. Thus, every proper subgroup of $A(\mathbb{Q})$ has infinite index in $A(\mathbb{Q})$.

Let $X \in \mathcal{N} 41$ be an infinite set. If $X$ is well-orderable in $\mathcal{N} 41$, then we have nothing to show. So assume that $X$ is not well-orderable in the model 
and let $E=\bigcup\left\{A_{n}: n<k+1\right\}$ (where $k \in \omega$ ) be a support of $X$. Then there exists an element $z$ of $X$ and a permutation $\phi \in \operatorname{fix}_{G}(E)$ such that $\phi(z) \neq z$. Let $E_{z}$ be a support of $z$. Without loss of generality, assume that $E_{z}=E \cup A_{k+1}$ and $\phi \in \operatorname{fix}_{G}\left(A \backslash A_{k+1}\right)$. Note that $\operatorname{fix}_{G}\left(A \backslash A_{k+1}\right)$ is isomorphic to $A\left(A_{k+1}\right)$ (i.e. the group of all order automorphisms of $\left(A_{k+1}, \leq_{k+1}\right)$ ). We denote $\operatorname{fix}_{G}\left(A \backslash A_{k+1}\right)$ by $\mathcal{G}$.

Let $Y=\{\psi(z): \psi \in \mathcal{G}\}$. Then $Y \subseteq X$ and $Y$ is well-orderable since $\operatorname{fix}_{G}\left(E_{z}\right) \subset \operatorname{fix}_{G}(Y)$. [Let $\pi \in \operatorname{fix}_{G}\left(E_{z}\right)$. Then for all $\psi \in \mathcal{G}$ and all $a \in E_{z}$ we have $\pi \psi(a)=\pi(\psi(a))=\psi(a)$, since $\psi(a) \in E_{z}$. Since $E_{z}$ is a support of $z$, and $\pi \psi, \psi$ agree on $E_{z}$, it follows that $\pi(\psi(z))=\psi(z)$; hence $\pi \in \operatorname{fix}_{G}(y)$.]

We assert that $Y$ is infinite. By way of contradiction, assume that $Y$ is finite, say $Y=\left\{z, \psi_{1}(z), \ldots, \psi_{m}(z)\right\}$ (so that $|Y|=m+1$ ). Note that $Y$ has at least two elements, since both $z$ and $\phi(z)$ are elements of $y$ and $\phi(z) \neq z$. It follows that the group

$$
H=\{\eta \in \mathcal{G}: \eta(z)=z\}
$$

is a proper subgroup of $\mathcal{G}$. Furthermore, the quotient group $\mathcal{G} / H$ is $\left\{H, \psi_{1} H, \ldots, \psi_{m} H\right\}$; hence the index $|\mathcal{G}: H|$ is $m+1$, and thus is finite. Since $\mathcal{G}$ is isomorphic to $A\left(A_{k+1}\right)$, it follows from Lemma 2 that there is a finite subset $U=\left\{u_{1}, \ldots, u_{r}\right\}$ of $A_{k+1}$ such that $H=\operatorname{fix}_{\mathcal{G}}(U)$. It is now fairly straightforward to construct a permutation $\pi \in \mathcal{G}$ such that for all $i<m+1, \pi^{-1} \psi_{i}$ does not fix $U$ pointwise ( $\psi_{0}$ is the identity mapping); hence $\pi^{-1} \psi_{i} \notin H$ for all $i<m+1$. It follows that $|\mathcal{G}: H|>m+1$, which is a contradiction. Thus, $Y$ is infinite, and hence $X$ is Dedekind-infinite as required. Therefore, DF $=\mathrm{F}$ is true in $\mathcal{N} 41$, and hence (by Theorem 3) so is "Every infinite set has an uncountable $\left(>\aleph_{0}\right)$ almost disjoint family".

Now since DF $=F$ is injectively boundable (see [5, Note 103]) and $\neg \mathrm{AC}_{\mathrm{DLO}}^{\aleph_{0}}$ is boundable, and hence injectively boundable, and $\Phi=\mathrm{DF}=\mathrm{F} \wedge$ $\neg \mathrm{AC}_{\mathrm{DLO}}^{\aleph_{0}}$ has a ZFA model, it follows from Pincus' transfer Theorem 4 in [9] that $\Phi$ has a ZF model in which "Every infinite set has an uncountable $\left(>\aleph_{0}\right)$ almost disjoint family" is true. This completes the proof of the theorem.

REMARK 2. In [5, Note 112], it is shown that every element $\psi \in A(\mathbb{Q})$ has an $n$th root for all $n \in \omega \backslash\{0\}$, that is, there is an element $\psi^{\prime} \in A(\mathbb{Q})$ such that $\left(\psi^{\prime}\right)^{n}=\psi$ (see [5, p. 294]). Using the above fact and essentially the same ideas as in the proof of the Lemma in [5. pp. 294-295] stating that $\mathrm{DF}=\mathrm{F}$ is true in $\mathcal{N} 41$, one easily obtains the key point for the proof, namely that every proper subgroup of $A(\mathbb{Q})$ has infinite index in $A(\mathbb{Q})$.

A variant of the model $\mathcal{N} 41$ which could also be used in order to establish Theorem 4 is as follows: The set $A$ of atoms is a denumerable disjoint union $A=\bigcup\left\{A_{n}: n \in \omega\right\}$, where each $A_{n}$ is ordered like the reals by $\leq_{n}$. The group $G$ and the normal filter $\Gamma$ of supports are defined as in the construction 
of $\mathcal{N} 41$. In the resulting model, say $\mathcal{N}, \mathrm{AC}_{\mathrm{DLO}}^{\aleph_{0}}$ is false, whereas $\mathrm{DF}=\mathrm{F}$ is true. The proof of the latter fact is almost identical to the corresponding one for $\mathcal{N} 41$; this time, one uses the result that the only subgroup of $A(\mathbb{R})$ (the group of order automorphisms of $\mathbb{R}$ with the usual ordering $\leq$ ) having index $<2^{\aleph_{0}}$ is $A(\mathbb{R})$ itself; thus, no proper subgroup of $A(\mathbb{R})$ can have index $<2^{\aleph_{0}}$ (see Truss [12] and Droste and Truss [2]).

THEOREM 5. The following hold:

(i) In ZFA, MC implies "For every infinite set $X$, every almost disjoint family in X can be extended to a MAD family in X". Hence, the latter statement does not imply $\mathrm{AC}_{\text {fin }}^{\aleph_{0}}$ in ZFA.

(ii) In ZF, PW implies "For every infinite set $X$, every almost disjoint family in $X$ can be extended to a MAD family in $X$ ".

(iii) PW does not imply "For every infinite set $X$, every almost disjoint family in $X$ can be extended to a MAD family in $X$ " in ZFA.

(iv) "For every infinite set $X$, every almost disjoint family in $X$ can be extended to a MAD family in $X$ " is not provable in ZF.

Proof. (i) Assume that $\mathrm{MC}$ is true. Let $X$ be an infinite set and also let $\mathcal{A}$ be an infinite almost disjoint family of infinite subsets of $X$. Assume that $\mathcal{A}$ is not a MAD family. Let $f$ be a multiple choice function of $\mathcal{P}(\mathcal{P}(X)) \backslash\{\emptyset\}$.

By transfinite recursion we define a MAD family of $X$ which contains $\mathcal{A}$. Let

$$
\mathcal{R}_{0}=\{Z \subseteq X: Z \text { is infinite and }|Z \cap A|<\omega \text { for all } A \in \mathcal{A}\} .
$$

Since $\mathcal{A}$ is not maximal, it follows that $\mathcal{R}_{0} \neq \emptyset$. Let

$$
R_{0}=\bigcup f\left(\mathcal{R}_{0}\right) \text {. }
$$

By the definition of $\mathcal{R}_{0}$, we see that $R_{0}$ is infinite, and we assert that $R_{0} \cap A$ is finite for all $A \in \mathcal{A}$. If not, then for some $A \in \mathcal{A}, R_{0} \cap A$ is infinite. Now, $R_{0} \cap A=\bigcup\left\{Z \cap A: Z \in f\left(\mathcal{R}_{0}\right)\right\}$ and since $R_{0} \cap A$ is infinite and $f\left(\mathcal{R}_{0}\right)$ is finite, it follows that for some $Z \in f\left(\mathcal{R}_{0}\right), Z \cap A$ is infinite. Furthermore, since $f\left(\mathcal{R}_{0}\right) \subseteq \mathcal{R}_{0}$, we have $Z \in \mathcal{R}_{0}$, and hence $Z \cap A$ is finite, which is a contradiction. Let

$$
\mathcal{A}_{0}=\mathcal{A} \cup\left\{R_{0}\right\} .
$$

Then $\mathcal{A}_{0}$ is almost disjoint. Now, assume that for some ordinal number $\alpha$ we have constructed an $\subseteq$-increasing sequence $\left(\mathcal{A}_{\beta}\right)_{\beta<\alpha}$ (where $\mathcal{A}_{0}=\mathcal{A} \cup\left\{R_{0}\right\}$ ) of almost disjoint families of infinite subsets of $X$. Let $\mathcal{B}_{\alpha}=\bigcup\left\{\mathcal{A}_{\beta}: \beta<\alpha\right\}$. Clearly, $\mathcal{B}_{\alpha}$ is an almost disjoint family of infinite subsets of $X$ which contains $\mathcal{A}$. If $\mathcal{B}_{\alpha}$ is maximal, then we are done. Otherwise, we may work as in the first step of the recursion, letting first

$$
\mathcal{R}_{\alpha}=\left\{Z \subseteq X: Z \text { is infinite and }|Z \cap B|<\omega \text { for all } B \in \mathcal{B}_{\alpha}\right\}
$$


(which is non-empty), and then letting

$$
\mathcal{A}_{\alpha}=\mathcal{B}_{\alpha} \cup\left\{R_{\alpha}\right\},
$$

where $R_{\alpha}=\bigcup f\left(\mathcal{R}_{\alpha}\right)$. Since the class of all ordinal numbers is a proper one, it follows that the recursion must terminate at some ordinal stage, say $\gamma$. By the above construction, this means that we have obtained a maximal almost disjoint family $\mathcal{A}_{\gamma}$ of infinite subsets of $X$ which contains $\mathcal{A}$.

The second assertion of (i) follows from the first one and the fact that in the Second Fraenkel Model, MC is true whereas $A C_{\text {fin }}^{\aleph_{0}}$ is false. This completes the proof of (i).

(ii) Assume that PW is true. Let $X$ be an infinite set and let $\mathcal{A}$ be an almost disjoint family in $X$. Since in ZF, PW is equivalent to AC, we know that $X$ is well-orderable, and hence by $\mathrm{PW}, \mathcal{P}(\mathcal{P}(X))$ is also well-orderable. Using the latter fact and following the proof of (i), we conclude that $\mathcal{A}$ can be extended to a MAD family in $X$. This completes the proof of (ii).

(iii) For the required independence result, we introduce an FM model, whose description is as follows: We start with a ground model $M$ of ZFA $+\mathrm{AC}$ with a set $A$ of atoms which is a denumerable disjoint union $A=$ $\bigcup\left\{A_{n}: n \in \omega\right\}$, where $\left|A_{n}\right|=\aleph_{0}$ for each $n \in \omega$. Let $G$ be the group of all permutations $\phi$ of $A$ such that $\phi\left(A_{n}\right)=A_{n}$ for each $n \in \omega$. Let $\mathcal{F}$ be the normal filter on $G$ which is generated by the pointwise stabilizers $\operatorname{fix}_{G}(E)$, where $E \subset A$ and $\left|E \cap A_{n}\right|<\aleph_{0}$ for all $n \in \omega$. Let $\mathcal{N}$ be the permutation model which is determined by $M, G$ and $\mathcal{F}$.

Note that PW is true in every FM model (see [5]), and hence PW is true in $\mathcal{N}$. Now, let $\mathcal{A}=\left\{A_{n}: n \in \omega\right\}$. Then $\mathcal{A}$ is denumerable in $\mathcal{N}$ since any permutation of $A$ in $G$ fixes $A_{n}$ for all $n \in \omega$, and any partial multiple choice function of $\mathcal{A}$ in the ground model $M$ is an element of $\mathcal{N}$ (since if $f$ is a partial multiple choice function of $\mathcal{A}$ in $M$, then $\operatorname{ran}(f)$ is a support of $f$ ). Furthermore, $\mathcal{A}$ is not a MAD family in $A$, since if $C$ is a choice set of $\mathcal{A}$, then $C \in \mathcal{N}, C \notin \mathcal{A}$ and $\mathcal{A} \cup\{C\} \in \mathcal{N}$ is an almost disjoint family in $A$. However, $\mathcal{A}$ cannot be extended to a MAD family in the model $\mathcal{N}$. Indeed, by way of contradiction assume that in $\mathcal{N}, \mathcal{A}$ can be extended to a MAD family in $A$, say $\mathcal{D}$. Then $\mathcal{A} \subsetneq \mathcal{D}$. Furthermore, $\mathcal{D} \backslash \mathcal{A}$ consists of partial multiple choice sets of $\mathcal{A}$ (i.e. multiple choice sets of infinite subsets of $\mathcal{A}$ ). Let $E \subset A$ be a support of $\mathcal{D}$. Note that $E$ cannot be finite. Indeed, otherwise, let $D$ be any element of $\mathcal{D} \backslash \mathcal{A}$ and also let $n \in \omega$ be such that $E \cap A_{n}=\emptyset$ and $D \cap A_{n} \neq \emptyset$. Let $a \in D \cap A_{n}$ and also let $b \in A_{n} \backslash D$ (recall that $A_{n} \cap D$ is finite). We consider the transposition $\eta=(a, b)$, i.e. $\eta$ swaps $a$ and $b$ and fixes all other atoms. Then $\eta \in \operatorname{fix}_{G}(E)$, and hence $\eta(\mathcal{D})=\mathcal{D}$. It follows that $\eta(D) \in \mathcal{D}$. However, $\eta(D) \neq D($ since $b=\eta(a) \in \eta(D) \backslash D)$ and $\eta(D) \cap D$ is infinite, contrary to $\mathcal{D}$ 's being almost disjoint. Therefore, $E$ is infinite as required. 
Using a similar argument to the one of the previous paragraph (and based on the fact that $\mathcal{D}$ is almost disjoint), we may conclude that for all $D \in \mathcal{D} \backslash \mathcal{A}$ we have $D \subset E$. But then, let $C$ be a choice set of $\mathcal{A}$ such that $C \cap E=\emptyset$. Clearly, $\mathcal{D} \cup\{C\}$ is in $\mathcal{N}$ and is an almost disjoint family in $A$ which properly contains $\mathcal{D}$, contrary to $\mathcal{D}$ 's being a MAD family in $A$. Thus, in $\mathcal{N}, \mathcal{A}$ cannot be extended to a MAD family, finishing the proof of (iii).

(iv) Note first that $\Psi=$ "There exists an infinite set $X$ and an almost disjoint family in $X$ which cannot be extended to a MAD family in $X$ " is a boundable statement (see Jech [6, Chapter 6] for the definition of the above term). By (iii), $\Psi$ has a permutation model of ZFA, and hence from the Jech-Sochor First Embedding Theorem (see [ $\underline{6}$, Theorem 6.1]), it follows that $\Psi$ has a symmetric model of ZF (see also [6, Problem 1 of Section 6.3]), finishing the proof of (iv) and of the theorem.

Recall that Törnquist [11 has shown that in Solovay's model (Model $\mathcal{M} 5(\aleph)$ in [5]), there are no infinite MAD families in $\omega$. This powerful result implies that "Every almost disjoint family in $\omega$ can be extended to a MAD family in $\omega$ " is not provable in ZF (and hence "For every infinite set $X$, every almost disjoint family in $X$ can be extended to a MAD family in $X$ " is not provable in ZF either). We should note here that the above statement is true in every Fraenkel-Mostowski permutation model of ZFA since $\omega$ is well-ordered and PW is true in every permutation model (see [5]).

Now in Theorem 5, we showed that in ZFA, MC implies "For every infinite set $X$, every almost disjoint family of infinite subsets of $X$ can be extended to a MAD family in $X$ ". Therefore, a natural question that emerges at this point is whether the above implication is reversible in ZFA. We will show that the answer is negative. Firstly, we need to prove the following lemma.

LEMma 3. The following statements are equivalent:

(i) For every infinite set $X$, every almost disjoint family in $X$ can be extended to a MAD family in $X$.

(ii) For every infinite set $X$, every almost disjoint family $\mathcal{A}$ in $X$ can be extended to a $M A D$ family in $\bigcup \mathcal{A}$.

Proof. (i) $\rightarrow$ (ii) Assume (i) holds. Let $X$ be an infinite set and let $\mathcal{A}$ be an almost disjoint family in $X$. By our hypothesis, let $\mathcal{C}$ be a MAD family in $X$ which contains $\mathcal{A}$. Let $\mathcal{C}^{\prime}$ be the trace of $\mathcal{C}$ in $\bigcup \mathcal{A}$, that is, $\mathcal{C}^{\prime}=\{C \cap \bigcup \mathcal{A}: C \in \mathcal{C}\}$. Then $\mathcal{C}^{\prime}$ is a MAD family in $\bigcup \mathcal{A}$ which contains $\mathcal{A}$. If $\mathcal{C}^{\prime}$ is not MAD in $\bigcup \mathcal{A}$, then let $Z \subset \cup \mathcal{A}$ be such that $Z \notin \mathcal{C}^{\prime}$ and $\mathcal{C}^{\prime} \cup\{Z\}$ is almost disjoint. Clearly, $Z \notin \mathcal{C}, \mathcal{C} \cup\{Z\}$ is almost disjoint in $X$ and contains $\mathcal{A}$, contrary to $\mathcal{C}$ 's being MAD in $X$. Thus, $\mathcal{C}^{\prime}$ is MAD in $\bigcup \mathcal{A}$ as required. 
(ii) $\rightarrow$ (i) Assume (ii) holds. Let $X$ be an infinite set and let $\mathcal{A}$ be an almost disjoint family in $X$. Let $\mathcal{C}$ be a MAD family in $\bigcup \mathcal{A}$ which contains $\mathcal{A}$. Two cases are possible:

CASE A: $X \backslash \cup \mathcal{A}$ is finite. Then

$$
\mathcal{C}^{\prime}=\{C \cup F: C \in \mathcal{C}, F \subseteq X \backslash \bigcup \mathcal{A}\}
$$

is a MAD family in $X$ which contains $\mathcal{A}$.

Case B: $X \backslash \cup \mathcal{A}$ is infinite. Then

$$
\mathcal{C}^{\prime \prime}=\left\{C \cup F: C \in \mathcal{C}, F \in[X \backslash \bigcup \mathcal{A}]^{<\omega}\right\} \cup\{X \backslash \bigcup \mathcal{A}\}
$$

is a MAD family in $X$ which contains $\mathcal{A}$. This completes the proof of (ii) $\rightarrow(\mathrm{i})$ and of the lemma.

THEOREM 6. "For every infinite set $X$, every almost disjoint family in $X$ can be extended to a MAD family in $X$ " does not imply MC in ZFA. In particular, the above statement is true in Mostowski's Linearly Ordered Model (Model $\mathcal{N} 3$ in [5]), in which MC is false.

Proof. (For the description of $\mathcal{N} 3$, see the proof of Theorem 2, It is known that (BPI is true in $\mathcal{N} 3$, whereas) $M C$ is false in $\mathcal{N} 3$ (see [5]). We also recall the following facts about the model $\mathcal{N} 3$ (see Jech [6, Section 4.5, pp. 49-51] for the details):

(1) $\mathcal{P}(A)$ is Dedekind-finite in $\mathcal{N} 3$.

(2) Every element $x \in \mathcal{N} 3$ has a least support, which we denote by $\operatorname{supp}(x)$. Furthermore, for each $x \in \mathcal{N} 3$ and each $\phi \in G, \phi(\operatorname{supp}(x))=$ $\operatorname{supp}(\phi(x))$ and $\phi(x)=x \Leftrightarrow \phi \in \operatorname{fix}_{G}(\operatorname{supp}(x))$. Yet, if $E \subset A$ is finite and $\phi \in G$, then $\phi(E)=E \Leftrightarrow \phi \in \operatorname{fix}_{G}(E)$.

(3) For every $x \in \mathcal{N} 3$, there is an ordinal number $\gamma$ and a one-to-one function $F: x \rightarrow \gamma \times[A]^{<\omega}$ which is in $\mathcal{N} 3$, and thus any $x \in \mathcal{N} 3$ can be written as a well-ordered disjoint union of Dedekind-finite sets. [If $x \in \mathcal{N} 3$, then we let $E \subset A$ be a finite support of $x$. For each $y \in x$, let $\operatorname{Orb}_{E}(y)$ be the orbit of $y$ under the action of the subgroup $\operatorname{fix}_{G}(E)$ of $G$, i.e. $\operatorname{Orb}_{E}(y)=$ $\left\{\phi(y): \phi \in \operatorname{fix}_{G}(E)\right\}$. Then the family $\mathcal{O}=\left\{\operatorname{Orb}_{E}(y): y \in x\right\}$ is well-ordered in $\mathcal{N} 3$ (since $E$ supports $\operatorname{Orb}_{E}(y)$ for all $y \in x$ ), and so we may let $\gamma$ be a well-ordered cardinal number and $f: \mathcal{O} \rightarrow \gamma$ be a bijection in $\mathcal{N} 3$. Define a function $F$ on $x$ by $F(y)=\left(f\left(\operatorname{Orb}_{E}(y)\right), \operatorname{supp}(y)\right)$ for all $y \in x$; then $F$ is a one-to-one function from $x$ into $\gamma \times[A]^{<\omega}$ which is in $\mathcal{N} 3$ since it has $E$ as a support; see also [6, Lemma 4.6].]

Now we turn to the proof that "For every infinite set $X$, every almost disjoint family of infinite subsets of $X$ can be extended to a MAD family in $X "$ is true in $\mathcal{N} 3$. To this end, let $X$ be an infinite set in $\mathcal{N} 3$ and let $\mathcal{A} \in \mathcal{N} 3$ be an almost disjoint family in $X$. We first note that if $X$ is well- 
orderable in $\mathcal{N} 3$, then by PW (which is true in every FM model), $\mathcal{A}$ can be extended to a MAD family in $X$ (see Theorem 5(ii)). So assume that $X$ is not well-orderable in $\mathcal{N} 3$. Our plan is to construct a MAD family (in $\mathcal{N} 3$ ) in $\cup \mathcal{A}$ which contains $\mathcal{A}$; then the required result will follow from Lemma 3.

Let $E \subset A$ be a finite support of $X$ and $\mathcal{A}$. By fact (3) above, we may view $X$ as a disjoint union $\bigcup\left\{X_{i}: i<\gamma\right\}$ (where $\gamma$ is a well-ordered cardinal number) such that for all $i<\gamma, X_{i} \subseteq[A]^{<\omega}$ (and hence-by fact (1)- $X_{i}$ is Dedekind-finite for all $i<\gamma$ ). Essentially, each $X_{i}$ corresponds to $\operatorname{Orb}_{E}(x)$ for some $x \in X$, and for that $x$, every element of $X_{i}$ is the least support $\operatorname{supp}(\phi(x))$ of $\phi(x)$ for some $\phi \in \operatorname{fix}_{G}(E)$. (Thus for each $i<\gamma$, there is $n(i) \in \omega$ such that $X_{i} \subseteq[A]^{n(i)}$.) By fact (1) and our assumption on $X$, we may assume (without loss of generality) that $X_{i}$ is infinite for all $i<\gamma$. We also make the following observations:

(a) Let $Z \in \mathcal{A}$. Since $\mathcal{P}(A)$ is Dedekind-finite (in $\mathcal{N} 3$ ) and $Z$ is infinite, it follows that $Z \cap X_{i}$ is non-empty and finite for only finitely many $i<\gamma$.

(b) For each $i<\gamma$, let

$$
\mathcal{W}_{i}=\left\{Z \cap X_{i}: Z \in \mathcal{A}\right\} .
$$

Then for all $i<\gamma, \mathcal{W}_{i} \in \mathcal{N} 3$ since it has $E$ as a support (recall that $E$ is a support of $\mathcal{A}$ and of $X_{i}$ for all $i<\gamma$ ). Furthermore, since $\mathcal{A}$ is almost disjoint, we have

$$
(\forall i<\gamma)\left(\forall Z \in \mathcal{W}_{i}\right)\left(\forall Z^{\prime} \in \mathcal{W}_{i}\right)\left(Z \neq Z^{\prime} \rightarrow\left|Z \cap Z^{\prime}\right|<\omega\right) .
$$

Without loss of generality, we assume that $\mathcal{W}_{i} \neq \emptyset$ for all $i<\gamma$. Since $\mathcal{P}(A)$ is Dedekind-finite, it follows that for only finitely many $i<\gamma$ is the set $\mathcal{W}_{i}^{*}=\left\{W \in \mathcal{W}_{i}: W\right.$ is finite $\}$ non-empty and finite. Without loss of generality, we may assume that $\mathcal{W}_{i}^{*}=\emptyset$ for all $i<\gamma$ (note that for each $i<\gamma$ such that $\mathcal{W}_{i}^{*}$ is non-empty and finite, we may readily augment $\mathcal{W}_{i}^{*}$ to a maximal family in $X_{i}$ such that any two distinct elements have finite intersection). Therefore, for all $i<\gamma$ and all $W \in \mathcal{W}_{i}, W$ is infinite. Moreover, it is not hard to verify that if for some $i<\gamma, \mathcal{W}_{i}$ is finite, then $\mathcal{W}_{i}$ can be (effectively) extended to a MAD family in $X_{i}$. Thus we further assume that for all $i<\gamma, \mathcal{W}_{i}$ is infinite and not MAD in $\bigcup \mathcal{W}_{i}$.

For each $i<\gamma$, by transfinite recursion on ordinals we will construct (in $\mathcal{N} 3$ ) a MAD family $\mathcal{B}_{i}$ in $\bigcup \mathcal{W}_{i}$. Then $\mathcal{A} \cup \bigcup\left\{\mathcal{B}_{i}: i<\gamma\right\}$ will be (in $\mathcal{N} 3$ ) the required MAD family in $\bigcup \mathcal{A}$ which contains $\mathcal{A}$. Fix $i<\gamma$. Since $\mathcal{W}_{i}$ is not MAD in $\bigcup \mathcal{W}_{i}$, there exists - in $\mathcal{N} 3$ - an infinite subset $B$ of $\bigcup \mathcal{W}_{i}$ such that $\mathcal{W}_{i} \cup\{B\}$ is an almost disjoint family which properly contains $\mathcal{W}_{i}$. In the ground model $M$, we let $\mathcal{Z}_{i}$ be the collection of all such infinite sets $B \in \mathcal{N} 3$. Since $\mathcal{Z}_{i} \subseteq \mathcal{P}\left(X_{i}\right)$ and $X_{i}$ is Dedekind-finite in $\mathcal{N} 3$, so is every $B \in \mathcal{Z}_{i}$ (and thus no element of $\mathcal{Z}_{i}$ is well-orderable in $\mathcal{N} 3$ ). Furthermore, it is easy to see that for every $B \in \mathcal{Z}_{i}, \psi(B) \cap W$ is finite for all $\psi \in \operatorname{fix}_{G}(E)$ 
and all $W \in \mathcal{W}_{i}$ (since for any $B \in \mathcal{Z}_{i}, \mathcal{W}_{i} \cup\{B\}$ is almost disjoint and $E$ is a support of $\mathcal{W}_{i}$.

In $M$ (which satisfies $\mathrm{AC}$ ), we let $\preceq$ be a well-ordering on $\mathcal{P}(X)$ (which clearly induces a well-ordering on $X)$. Recall also that $\leq$ is a linear order on $A$ in $\mathcal{N} 3$, and thus $[A]^{<\omega} \backslash\{\emptyset\}$ has a choice function.

For the base step of the induction, consider any $B_{0} \in \mathcal{Z}_{i}$. Let $E_{0}=$ $\operatorname{supp}\left(B_{0}\right)$. Then $E \cup E_{0}$ is also a support of $B_{0}$, and since $B_{0}$ is not wellorderable in $\mathcal{N} 3$, there is a $\preceq$-least $t^{*} \in B_{0}$ such that $\operatorname{supp}\left(t^{*}\right) \backslash\left(E \cup E_{0}\right)$ $\neq \emptyset\left({ }^{1}\right)$. Let $a^{*}$ be the $\leq$-least element of $\operatorname{supp}\left(t^{*}\right) \backslash\left(E \cup E_{0}\right)$, and also let $I=E \cup\left(\operatorname{supp}\left(t^{*}\right) \backslash\left\{a^{*}\right\}\right)$ and assume $I=\left\{a_{1}, \ldots, a_{n}\right\}$, where $a_{1}<\cdots<a_{n}$. If we let $a_{0}=-\infty$ and $a_{n+1}=+\infty$, then the atom $a^{*}$ occurs in one of the intervals $\left(a_{j}, a_{j+1}\right)$ where $0 \leq j \leq n$. Note that there is exactly one element of $\operatorname{supp}\left(t^{*}\right)$ in $\left(a_{j}, a_{j+1}\right)$ (namely $\left.a^{*}\right)$ and $\left(a_{j}, a_{j+1}\right) \cap E=\emptyset$. Now we let

$$
f=\left\{\left(\phi\left(a^{*}\right), \phi\left(t^{*}\right)\right): \phi \in \operatorname{fix}_{G}(I)\right\} .
$$

Working exactly as in the proof of the following known fact about $\mathcal{N} 3$ : "If a set in $\mathcal{N} 3$ is not well-orderable, then it contains an infinite subset which has the same cardinality as some open interval $(a, b) \subset A$ (in the linear ordering of $A$ in $\mathcal{N} 3$ )" (see Brunner [1]), we may conclude that $f \in \mathcal{N} 3$ (since $I$ is a support of $f) ; f$ is a function with domain the open interval $\left(a_{j}, a_{j+1}\right) ; f$ is one-to-one (this uses fact (2)); and the range of $f, C_{0}=\left\{\phi\left(t^{*}\right): \phi \in \operatorname{fix}_{G}(I)\right\}$, is in $\mathcal{N} 3$ (since $I$ is a support of $C_{0}$ ), and is infinite since $\left|C_{0}\right|=\left|\left(a_{j}, a_{j+1}\right)\right|$. Furthermore, $C_{0} \subseteq \bigcup \mathcal{W}_{i}$ since $t^{*} \in B_{0} \subseteq \bigcup \mathcal{W}_{i}$ and $I$ contains the support $E$ of $\mathcal{W}_{i}$ (specifically, $t^{*} \in B_{0}$ implies that $t^{*} \in W$ for some $W \in \mathcal{W}_{i}$ (since $\left.B_{0} \subseteq \bigcup \mathcal{W}_{i}\right)$, hence for each $\phi \in$ fix $_{G}(I)$, we have $\phi\left(t^{*}\right) \in \phi(W)$ and $\phi(W)$ is in $\phi\left(\mathcal{W}_{i}\right)=\mathcal{W}_{i}$, since $\left.E \subset I\right)$. We also note that for any $\phi \in \operatorname{fix}_{G}(I)$,

$$
\begin{aligned}
\operatorname{supp}\left(\phi\left(t^{*}\right)\right) & =\phi\left(\operatorname{supp}\left(t^{*}\right)\right)=\phi\left(\left(\operatorname{supp}\left(t^{*}\right) \backslash\left\{a^{*}\right\}\right) \cup\left\{a^{*}\right\}\right) \\
& =\operatorname{supp}\left(t^{*}\right) \backslash\left\{a^{*}\right\} \cup\left\{\phi\left(a^{*}\right)\right\} .
\end{aligned}
$$

By (1), for any $\rho \in G$ and $\phi\left(t^{*}\right) \in C_{0}$, if $\rho\left(\operatorname{supp}\left(t^{*}\right) \backslash\left\{a^{*}\right\}\right) \neq \operatorname{supp}\left(t^{*}\right) \backslash\left\{a^{*}\right\}$ then $\rho\left(\phi\left(t^{*}\right)\right) \notin C_{0}$, and thus $\rho\left(C_{0}\right) \cap C_{0}=\emptyset$. Now if $\rho \in \operatorname{fix}_{G}(E)$ and $\rho(I) \neq I$, then $($ since $\rho(E)=E) \rho\left(\operatorname{supp}\left(t^{*}\right) \backslash\left\{a^{*}\right\}\right) \neq \operatorname{supp}\left(t^{*}\right) \backslash\left\{a^{*}\right\}$. As noted above, this yields $\rho\left(C_{0}\right) \cap C_{0}=\emptyset$. Thus, we have shown that for all $\rho$ in $\operatorname{fix}_{G}(E)$ either $\rho\left(C_{0}\right)=C_{0}$ or $C_{0} \cap \rho\left(C_{0}\right)=\emptyset$. Hence $\left\{\rho\left(C_{0}\right): \rho \in \operatorname{fix}_{G}(E)\right\}$ is disjoint and belongs to $\mathcal{N} 3$ (since it is supported by $E$ ). Now we prove that $\mathcal{W}_{i} \cup\left\{\rho\left(C_{0}\right): \rho \in \operatorname{fix}_{G}(E)\right\}$ is almost disjoint. In view of the above observations, it suffices to show that $\mathcal{W}_{i} \cup\left\{C_{0}\right\}$ is almost disjoint. Assume on the contrary that for some $W \in \mathcal{W}_{i}, W \cap C_{0}$ is infinite. Then for some $d, d^{\prime} \in\left(a_{j}, a_{j+1}\right)$ with $d<d^{\prime}$ we have $f\left(\left(d, d^{\prime}\right)\right) \subseteq W \cap C_{0}$. Indeed, since

$\left({ }^{1}\right)$ If $\mathcal{V}$ is an FM model which is determined by a ground model $M$, a group $G$ of permutations of the set $A$ of atoms, and a normal filter $\mathcal{F}$ of subgroups of $G$, then an element $x$ of $\mathcal{V}$ can be well-ordered in $\mathcal{V}$ if and only if $\operatorname{fix}_{G}(x) \in \mathcal{F}$ (see [6, p. 47]). 
$W \cap C_{0}$ is infinite, there is a $\phi \in \operatorname{fix}_{G}(I)$ such that $\phi\left(a^{*}\right) \notin \operatorname{supp}(W)$ and $\phi\left(t^{*}\right) \in W \cap C_{0}$. Let $d, d^{\prime}$ with $a_{j}<d<d^{\prime}<a_{j+1}, \phi\left(a^{*}\right) \in\left(d, d^{\prime}\right)$, and $\left(d, d^{\prime}\right) \cap \operatorname{supp}(W)=\emptyset$. Then

$$
g=\left\{\left(\pi\left(\phi a^{*}\right), \pi\left(\phi t^{*}\right)\right): \pi \in \operatorname{fix}_{G}\left(I \cup \operatorname{supp}(W) \cup\left\{d, d^{\prime}\right\}\right)\right\} \subseteq f,
$$

and so $f\left(\left(d, d^{\prime}\right)\right)=\operatorname{ran}(g) \subseteq W \cap C_{0}$.

A similar argument shows that $C_{0}$ contains an infinite subset of $B_{0}$. Indeed, since $a^{*} \notin E_{0}$, there is a subinterval $\left(s, s^{\prime}\right) \subseteq\left(a_{j}, a_{j+1}\right)$ such that $a^{*} \in\left(s, s^{\prime}\right)$ and $\left(s, s^{\prime}\right) \cap E_{0}=\emptyset$. Then

$$
h=\left\{\left(\psi\left(a^{*}\right), \psi\left(t^{*}\right)\right): \psi \in \operatorname{fix}_{G}\left(I \cup E_{0} \cup\left\{s, s^{\prime}\right\}\right)\right\} \subseteq f,
$$

and so $f\left(\left(s, s^{\prime}\right)\right)=\operatorname{ran}(h) \subseteq B_{0}$.

Now we consider an order automorphism $\gamma$ of $A$ such that $\gamma \in \operatorname{fix}_{G}(E)$ and $\gamma(s)<d<d^{\prime}<\gamma\left(s^{\prime}\right)$ (which is possible since $s, s^{\prime}, d$ and $d^{\prime}$ are all in $\left(a_{j}, a_{j+1}\right), s<s^{\prime}, d<d^{\prime}$ and $\left.\left(a_{j}, a_{j+1}\right) \cap E=\emptyset\right)$. Then we have $f\left(\left(d, d^{\prime}\right)\right) \subseteq$ $f\left(\left(\gamma(s), \gamma\left(s^{\prime}\right)\right)\right)=f\left(\gamma\left(\left(s, s^{\prime}\right)\right)\right)=\gamma\left(f\left(\left(s, s^{\prime}\right)\right)\right) \subseteq \gamma\left(B_{0}\right)$, and thus $\gamma\left(B_{0}\right) \cap W$ is infinite, which is a contradiction (see the discussion before the beginning of the induction). Thus $W \cap C_{0}$ is finite as required.

Let $\mathcal{V}_{i, 0}=\mathcal{W}_{i} \cup\left\{\rho\left(C_{0}\right): \rho \in \operatorname{fix}_{G}(E)\right\}$. Note that for each $\rho \in \operatorname{fix}_{G}(E)$, $\rho\left(C_{0}\right) \subseteq \bigcup \mathcal{W}_{i}$, and $\mathcal{V}_{i, 0} \in \mathcal{N} 3$ since it is supported by $E$. Furthermore, $\mathcal{V}_{i, 0}$ is almost disjoint.

Assume that for some ordinal number $\alpha$, we have defined a strictly $\subseteq$ increasing sequence $\left(\mathcal{V}_{i, \beta}\right)_{\beta<\alpha}$ of almost disjoint families in $\bigcup \mathcal{W}_{i}$, each having $E$ as a support (and properly extending $\mathcal{W}_{i}$ ). Let $\mathcal{R}_{i, \alpha}=\bigcup\left\{\mathcal{V}_{i, \beta}: \beta<\alpha\right\}$. (Clearly $\mathcal{R}_{i, \alpha}$ is almost disjoint in $\bigcup \mathcal{W}_{i}$ and has $E$ as a support). If $\mathcal{R}_{i, \alpha}$ is MAD in $\bigcup \mathcal{W}_{i}$, then the induction terminates. Otherwise, we may let $B_{\alpha}$ be the $\preceq$-least element of $\mathcal{Z}_{i}$ such that $B_{\alpha} \notin \mathcal{R}_{i, \alpha}$ and $\mathcal{R}_{i, \alpha} \cup\left\{B_{\alpha}\right\}$ is almost disjoint in $\bigcup \mathcal{W}_{i}$. Then working as in the base step of the induction, we may properly extend $\mathcal{R}_{i, \alpha}$ to a family $\mathcal{V}_{i, \alpha}=\mathcal{R}_{i, \alpha} \cup\left\{\rho\left(C_{\alpha}\right): \rho \in \operatorname{fix}_{G}(E)\right\}$ which is almost disjoint in $\bigcup \mathcal{W}_{i}$ and has $E$ as a support.

Since the class Ord of all ordinal numbers is a proper one, it follows that the recursion must terminate at some ordinal stage. By the above construction, this means that at this stage we have ended up with a MAD family $\mathcal{B}_{i}$ in $\bigcup \mathcal{W}_{i}$, which has $E$ as a support.

Now we let $\mathcal{B}=\mathcal{A} \cup \bigcup\left\{\mathcal{B}_{i}: i<\gamma\right\}$. Then $\mathcal{B} \in \mathcal{N} 3(E$ is a support of $\mathcal{B})$ and is a MAD family in $\bigcup \mathcal{A}$ which contains $\mathcal{A}$. By Lemma 3, we conclude now that $\mathcal{A}$ can be extended to a MAD family in $X$ as required.

Remark 3. Let $X$ be an infinite set and let $\mathcal{P}_{\infty}(X)$ be the set of infinite subsets of $X$. Let $\subseteq^{*}$ be the binary relation on $\mathcal{P}_{\infty}(X)$ defined by $A \subseteq^{*} B \Longleftrightarrow A \backslash B$ is finite and $B \backslash A$ is infinite. Then $\subseteq^{*}$ is a partial order order on $\mathcal{P}_{\infty}(X)$. Furthermore, if $\mathcal{A} \subseteq \mathcal{P}_{\infty}(X)$, 
then $\mathcal{A}$ is almost disjoint in $X$ if and only if $\mathcal{A}$ is an antichain in $\mathcal{P}_{\infty}(X)$ (i.e. any two distinct elements of $\mathcal{A}$ are incompatible with respect to $\subseteq^{*}$; if $A, B \in \mathcal{A}$ with $A \neq B$, then there exists no $D \in \mathcal{P}_{\infty}(X)$ such that $D \subseteq \subseteq^{*} A$ and $\left.D \subseteq * B\right)$. Thus, the statement "For every infinite set $X$, every almost disjoint family in $X$ can be extended to a MAD family in $X^{\prime \prime}$ can be reformulated as

"For every infinite set $X$, every antichain in the poset $\left(\mathcal{P}_{\infty}(X), \subseteq^{*}\right)$ can be extended to a maximal antichain."

By Theorems 5 and 6, the latter statement is strictly weaker than MC in ZFA. Furthermore, we note that

"Every poset has a maximal antichain" is equivalent to AC in ZFA.

Indeed, let $\mathcal{A}$ be a family of pairwise disjoint non-empty sets. Let $P=$ $\bigcup\{\mathbb{R} \times X: X \in \mathcal{A}\}$ and let $\prec$ be the following binary relation on $P$ :

$(r, x) \prec(s, y) \Longleftrightarrow r<s$ and there exists $X \in \mathcal{A}$ such that $x, y \in X$,

where $<$ is the usual order on $\mathbb{R}$. Then $\prec \cup\{((r, x),(r, x)): r \in \mathbb{R}, x \in \bigcup \mathcal{A}\}$ is a partial order on $P$, and hence there exists a maximal set $A \subseteq P$ of pairwise incompatible elements. By the definition of $\prec$, it easily follows that $A \cap(\mathbb{R} \times X) \neq \emptyset$ for all $X \in \mathcal{A}$. We assert that $A \cap(\mathbb{R} \times X)$ is a singleton, for each $X \in \mathcal{A}$. Towards a contradiction, assume that for some $X \in \mathcal{A}$, $(r, x),(s, y) \in A \cap(\mathbb{R} \times X)$ with $(r, x) \neq(s, y)$. Since the elements of $A$ are pairwise incompatible, we must have $r=s$, and thus $x \neq y$. Let $t \in \mathbb{R}$ with $t<r$. Then $(t, x) \prec(r, x)$ and $(t, x) \prec(s, y)$. This is a contradiction; hence $x=y$. It follows that the function $f$ on $\mathcal{A}$ defined by

$f(X)=$ the second coordinate of the unique ordered pair in $A \cap(\mathbb{R} \times X)$ is a choice function of $\mathcal{A}$.

We also recall that the statement "Every poset has a maximal set of pairwise incomparable elements" is equivalent to AC in ZF (see [6. Theorem 9.1]), but not equivalent to AC in ZFA; in particular, the above statement is true in the Basic Fraenkel Model $\mathcal{N} 1$ of [5] (see [6, Theorem 9.2]).

It is a well-known ZF-result that no MAD family in $\omega$ is countable. However, in the general case where $\omega$ is replaced by any infinite set $X$, the situation is strikingly different, so that the resulting statement is actually equivalent to a weak form of $\mathrm{AC}$, as the following theorem clarifies.

THEOREM 7. The following statements are equivalent:

(i) $\mathrm{MC}^{\aleph_{0}}$.

(ii) For every infinite set $X$, no infinite $M A D$ family in $X$ has cardinality $\aleph_{0}$.

Proof. (i) $\rightarrow$ (ii) Assume that $\mathrm{MC}^{\aleph_{0}}$ is true. Let $X$ be an infinite set. By way of contradiction, assume that $X$ has a MAD family $\mathcal{A}=\left\{A_{n}: n \in \omega\right\}$ 
of cardinality $\aleph_{0}$ (the mapping $n \mapsto A_{n}, n \in \omega$, is a bijection). Let $B_{0}=A_{0}$ and for $n \in \omega \backslash\{0\}$, let $B_{n}=A_{n} \backslash \bigcup\left\{A_{m}: m<n\right\}$. Since $\mathcal{A}$ is almost disjoint, $B_{n}$ is infinite for all $n \in \omega$. By $\mathrm{MC}^{\aleph_{0}}$, let $f$ be a multiple choice function of the family $\mathcal{B}=\left\{B_{n}: n \in \omega\right\}$. Then $C=\bigcup\{f(n): n \in \omega\}$ is an infinite subset of $X$ such that $C \notin \mathcal{A}$ and $C \cap A_{n}$ is finite for all $n \in \omega$, contrary to $\mathcal{A}$ 's being a MAD family.

(ii) $\rightarrow$ (i) Assume that (ii) is true. Since $M C^{\aleph_{0}}$ is equivalent to $P M C^{\aleph_{0}}$ ("Every countably infinite set of non-empty sets has a partial multiple choice function"), it suffices to prove that $\mathrm{PMC}^{\aleph_{0}}$ is true. Towards a contradiction, assume that there exists a denumerable family $\mathcal{A}=\left\{A_{n}: n \in \omega\right\}$ of nonempty sets without a partial multiple choice function. Without loss of generality, we assume that $\mathcal{A}$ is disjoint and each $A_{n}$ is infinite. Let $X=\bigcup \mathcal{A}$. Since $\mathcal{A}$ has no partial multiple choice function, it readily follows that $\mathcal{A}$ is a MAD family in $X$ of cardinality $\aleph_{0}$, contrary to our assumption. This completes the proof of the implication and of the theorem.

REMARK 4. It is known (see [5]) that $M C^{\aleph_{0}}$ is false in the Basic Fraenkel Model (Model $\mathcal{N} 1$ in [5]). To illustrate this, using the result of Theorem 7 above, let $A$ be the set of atoms of $\mathcal{N} 1$. It is known that $A$ is amorphous in $\mathcal{N} 1$ (see [5], [6]). Let $X=[A]^{<\omega}$ (the set of finite subsets of $A$ ) and also let $\mathcal{A}=\left\{[A]^{n}: n \in \omega \backslash\{0\}\right\}$ (where $[A]^{n}$ is the set of $n$-element subsets of $A$ ). Then $\mathcal{A}$ is a disjoint family of infinite subsets of $X$ and is countably infinite in $\mathcal{N} 1$ ( $\emptyset$ is a support of each element of $\mathcal{A})$. Furthermore, $\mathcal{A}$ is a MAD family in $X$. (Otherwise there is an infinite set $z \subseteq X$ in $\mathcal{N} 1$ such that $z \cap[A]^{n}$ is finite for all $n \in \omega \backslash\{\emptyset\}$; however, $z$ cannot be supported by any finite subset of $A$, as can be easily checked.)

Next, we provide a characterization of $D F=F$ in the language of almost disjoint families of functions.

THEOREM 8. The following statements are equivalent:

(i) $\mathrm{DF}=\mathrm{F}$.

(ii) For every infinite set $A$, there is a continuum sized almost disjoint family $\mathcal{A} \subseteq A^{\omega}$ (i.e. for any distinct $f, g \in \mathcal{A}$, the set $\{n \in \omega: f(n)=g(n)\}$ is finite).

Proof. (i) $\rightarrow$ (ii) Assume that DF $=\mathrm{F}$ is true. Let $A$ be an infinite set. By $\mathrm{DF}=\mathrm{F}$, let $D$ be a countably infinite subset of $A$, which we identify with $\mathbb{Q}$. For every $a \in \mathbb{R}$, choose a function $f_{a} \in \mathbb{Q}^{\omega}$ such that $\lim _{n \rightarrow \infty} f_{a}(n)=a$. Then letting $\mathcal{A}=\left\{f_{a}: a \in \mathbb{R}\right\}$, we have $\mathcal{A} \subseteq A^{\omega},|\mathcal{A}|=2^{\aleph_{0}}$ and for any distinct reals $a$ and $a^{\prime}$, the set $\left\{n \in \omega: f_{a}(n)=f_{a^{\prime}}(n)\right\}$ is finite; thus $\mathcal{A}$ is almost disjoint.

(ii) $\rightarrow$ (i) We first prove the following auxiliary result. 
LemMA 4. "For every infinite set $A$, there is a continuum sized almost disjoint family $\mathcal{A} \subseteq A^{\omega} "$ implies $\mathrm{AC}_{\text {fin }}^{\aleph_{0}}$.

Proof. Assume the hypothesis holds. Let $\mathcal{Z}=\left\{Z_{n}: n \in \omega\right\}$ be a countably infinite disjoint family of non-empty finite sets. Towards a contradiction, assume that $\mathcal{Z}$ has no partial choice function. (Recall that $\mathrm{AC}_{\text {fin }}^{\aleph_{0}}$ is equivalent to its partial version $\mathrm{PAC}_{\text {fin }}^{\aleph_{0}}$.) Let $A=\bigcup \mathcal{Z}$. By our hypothesis, there is a continuum sized almost disjoint family $\mathcal{A}=\left\{f_{r}: r \in \mathbb{R}\right\} \subseteq A^{\omega}$. Let $T=\left\{f_{n}: n \in \omega\right\}$; then $T$ is almost disjoint. Since $\mathcal{Z}$ has no partial choice function, for every $n \in \omega$ there exists a finite set $M_{n} \subset \omega$ such that

$$
m \in M_{n} \Longleftrightarrow \operatorname{ran}\left(f_{n}\right) \cap Z_{m} \neq \emptyset \text {. }
$$

Furthermore, since $T$ is almost disjoint and $\left|Z_{n}\right|<\omega$ for all $n \in \omega$, the set $\mathcal{M}=\left\{M_{n}: n \in \omega\right\}$ is infinite.

As $\mathcal{M}$ is infinite, via an easy induction we may construct a partial choice function of $\mathcal{Z}$, contrary to our assumption on $\mathcal{Z}$. We leave the easy details to the reader.

Now we return to the proof of (ii) $\rightarrow$ (i) in Theorem 8. Assume (ii) holds. For contradiction, assume that there exists an infinite Dedekind-finite set, say $D$. For each $n \in \omega$, let

$$
D_{n}=\left\{f \in D^{n}: f \text { is one-to-one }\right\} .
$$

Then each $D_{n}$ is Dedekind-finite, and hence so is $A=\bigcup\left\{D_{n}: n \in \omega\right\}$. Let $\mathcal{A}=\left\{f_{r}: r \in \mathbb{R}\right\} \subseteq A^{\omega}$ be a continuum sized almost disjoint family. Since $A$ is Dedekind-finite, it follows that for all $r \in \mathbb{R}, \operatorname{ran}\left(f_{r}\right)$ is finite, and since $\mathcal{A}$ is almost disjoint, $\left\{\operatorname{ran}\left(f_{r}\right): r \in \mathbb{R}\right\}$ is infinite; in particular, the set $\left\{\operatorname{ran}\left(f_{n}\right): n \in \omega\right\}$ is infinite. By Lemma 4, $\mathrm{AC}_{\text {fin }}^{\aleph_{0}}$ is true, and thus $W=\bigcup\left\{\operatorname{ran}\left(f_{n}\right): n \in \omega\right\}$ is a countably infinite subset of $A$, contrary to $A$ 's being Dedekind-finite. Thus, $D$ is Dedekind-infinite as required. The proof of the implication and of the theorem is complete.

COROllary 1. DF = F implies "For every free ultrafilter $\mathcal{U}$ on $\omega$ and every infinite set $A,\left|A^{\omega} / \mathcal{U}\right| \geq 2^{\aleph_{0}} "$.

Proof. Assume that DF $=\mathrm{F}$ is true. Let $\mathcal{U}$ be a free ultrafilter on $\omega$ and let $A$ be an infinite set. By Theorem 8 , there exists a continuum sized almost disjoint family $\mathcal{A}=\left\{f_{r}: r \in \mathbb{R}\right\} \subseteq A^{\omega}$. Therefore, for any distinct $r, r^{\prime} \in \mathbb{R}$ the set $\left\{n \in \omega: f_{r}(n)=f_{r^{\prime}}(n)\right\}$ is finite. Since $\mathcal{U}$ is a free ultrafilter, for distinct reals $r, r^{\prime}$ we have $\left\{n \in \omega: f_{r}(n)=f_{r^{\prime}}(n)\right\} \notin \mathcal{U}$, and thus $f_{r} \nsim_{\mathcal{U}} f_{r^{\prime}}$ (recall that $f \sim_{\mathcal{U}} g \Leftrightarrow\{n \in \omega: f(n)=g(n)\} \in \mathcal{U}$ ), so $\left[f_{r}\right] \cap\left[f_{r^{\prime}}\right]=\emptyset$. Hence, $\left|\left\{\left[f_{r}\right]: r \in \mathbb{R}\right\}\right|=2^{\aleph_{0}}$, and consequently $\left|A^{\omega} / \mathcal{U}\right| \geq 2^{\aleph_{0}}$.

THEOREM 9. Let $\mathcal{U}$ be a free ultrafilter on $\omega$ and let $A$ be an infinite set. If $\left|A^{\omega} / \mathcal{U}\right| \geq 2^{\aleph_{0}}$, then $A$ is Dedekind-infinite. 
Proof. Towards a contradiction, assume that $A$ is Dedekind-finite. Then $|\operatorname{ran}(f)|<\aleph_{0}$ for all $f \in A^{\omega}$. Fix temporarily $f$ in $A^{\omega}$. Since $\omega=\bigcup\left\{f^{-1}(\{a\})\right.$ : $a \in \operatorname{ran}(f)\} \in \mathcal{U}$ and $\mathcal{U}$ is an ultrafilter on $\omega$, there is a unique $a \in \operatorname{ran}(f)$ such that $f^{-1}(\{a\}) \in \mathcal{U}$. Let $g \in[f](=$ the $\sim \mathcal{U}$ equivalence class of $f)$. We assert that $g^{-1}(\{a\}) \in \mathcal{U}$. If not, then $\{n \in \omega: g(n) \neq a\}=\left(g^{-1}(\{a\})\right)^{c} \in \mathcal{U}$ (since $\mathcal{U}$ is an ultrafilter). Let

$$
Z=f^{-1}(\{a\}) \cap\{n \in \omega: f(n)=g(n)\} .
$$

Then $Z \in \mathcal{U}$, since $f^{-1}(\{a\}) \in \mathcal{U},\{n \in \omega: f(n)=g(n)\} \in \mathcal{U}$ (since $g \in[f]$ ) and $\mathcal{U}$ is a filter. Since $Z \subseteq g^{-1}(\{a\})$, it follows that $g^{-1}(\{a\}) \in \mathcal{U}$, which is a contradiction. Thus, $g^{-1}(\{a\}) \in \mathcal{U}$ as asserted.

From the above arguments, it follows that

$$
\left(\forall Y \in A^{\omega} / \mathcal{U}\right)\left(\exists ! a_{Y} \in A\right)(\forall f \in Y)\left(f^{-1}\left(\left\{a_{Y}\right\}\right) \in \mathcal{U}\right) .
$$

Furthermore, if $Y, Y^{\prime} \in A^{\omega} / \mathcal{U}$ with $Y \neq Y^{\prime}$ (and thus $Y \cap Y^{\prime}=\emptyset$ ), then $a_{Y} \neq a_{Y^{\prime}}$. Indeed, assume the contrary; then there exist distinct elements $Y=[f]$ and $Y^{\prime}=\left[f^{\prime}\right]$ of $A^{\omega} / \mathcal{U}$ such that $a_{Y}=a_{Y^{\prime}}$. Since $Y \neq Y^{\prime}$, we have $f \nsim_{\mathcal{U}} f^{\prime}$, and thus $\left\{n \in \omega: f(n)=f^{\prime}(n)\right\} \notin \mathcal{U}$. By (2), we have $f^{-1}\left(\left\{a_{Y}\right\}\right) \in \mathcal{U}$ and $\left(f^{\prime}\right)^{-1}\left(\left\{a_{Y^{\prime}}\right\}\right)=\left(f^{\prime}\right)^{-1}\left(\left\{a_{Y}\right\}\right) \in \mathcal{U}$, and since $\mathcal{U}$ is a filter, it follows that $W:=f^{-1}\left(\left\{a_{Y}\right\}\right) \cap\left(f^{\prime}\right)^{-1}\left(\left\{a_{Y}\right\}\right) \in \mathcal{U}$. Since $W \subseteq\{n \in \omega$ : $\left.f(n)=f^{\prime}(n)\right\}$, we conclude that $\left\{n \in \omega: f(n)=f^{\prime}(n)\right\} \in \mathcal{U}$. This is a contradiction; thus $a_{Y} \neq a_{Y^{\prime}}$ as required.

Therefore, the mapping $F: A^{\omega} / \mathcal{U} \rightarrow A$ defined by $F(Y)=a_{Y}$ is (welldefined and) one-to-one, and thus $\left|A^{\omega} / \mathcal{U}\right| \leq|A|$. By our hypothesis, we have $2^{\aleph_{0}} \leq\left|A^{\omega} / \mathcal{U}\right|$, and so $2^{\aleph_{0}} \leq|A|$. But then $A$ is Dedekind-infinite, which is a contradiction. This completes the proof of the theorem.

THEOREM 10. The following hold:

(i) $\operatorname{UF}(\omega)+$ "For every free ultrafilter $\mathcal{U}$ on $\omega$ and every infinite set $A$, $\left|A^{\omega} / \mathcal{U}\right| \geq 2^{\aleph_{0}}$ " implies DF $=\mathrm{F}$. It follows that "There exists a free ultrafilter $\mathcal{U}$ on $\omega$ and an infinite set $A$ such that $\left|A^{\omega} / \mathcal{U}\right| \nsupseteq 2^{\aleph_{0}} "$ is relatively consistent with ZF.

(ii) $\operatorname{UF}(\omega)+$ "For every free ultrafilter $\mathcal{U}$ on $\omega$ and every infinite set $A$, $\left|A^{\omega} / \mathcal{U}\right| \geq 2^{\aleph_{0}}$ " is equivalent to $\mathrm{UF}(\omega)+\mathrm{DF}=\mathrm{F}$. Hence, in every Fraenkel-Mostowski model of ZFA, "For every free ultrafilter $\mathcal{U}$ on $\omega$ and every infinite set $A,\left|A^{\omega} / \mathcal{U}\right| \geq 2^{\aleph_{0}}$ " is equivalent to $\mathrm{DF}=\mathrm{F}$.

(iii) "For every free ultrafilter $\mathcal{U}$ on $\omega$ and every infinite set $A,\left|A^{\omega} / \mathcal{U}\right| \geq 2^{\aleph_{0}}$ " does not imply $\mathrm{DF}=\mathrm{F}$ in $\mathrm{ZF}$.

(iv) MC does not imply "For every ultrafilter $\mathcal{U}$ on $\omega$ and every infinite set $A$, $\left|A^{\omega} / \mathcal{U}\right| \geq 2^{\aleph_{0}}$ " in ZFA.

(v) BPI does not imply "For every free ultrafilter $\mathcal{U}$ on $\omega$ and every infinite set $A,\left|A^{\omega} / \mathcal{U}\right| \geq 2^{\aleph_{0}}$ " in ZF. 
Proof. (i) The first assertion follows from Theorem 9. For the second assertion, it is known that in the Basic Cohen Model (Model $\mathcal{M} 1$ in [5]), BPI (and thus $\operatorname{UF}(\omega)$ ) is true, whereas DF $=\mathrm{F}$ is false; hence, "For every ultrafilter $\mathcal{U}$ on $\omega$ and for every infinite set $A,\left|A^{\omega} / \mathcal{U}\right| \geq 2^{\aleph_{0}}$ " is also false.

(ii) The first equivalence follows from Corollary 1 and Theorem 9 . The second assertion follows from the first and the fact that in every permutation model, $\operatorname{UF}(\omega)$ is true (see [5]).

(iii) The statement "For every ultrafilter $\mathcal{U}$ on $\omega$ and every infinite set $A,\left|A^{\omega} / \mathcal{U}\right| \geq 2^{\aleph_{0}}$ " is vacuously true in Blass' model $\mathcal{M} 15$ of [5], since all ultrafilters (on any set) are principal in $\mathcal{M} 15$. On the other hand, Tachtsis [10] has shown that in $\mathcal{M} 15$ there exists a countably infinite family of pairs which has no partial choice function, and thus DF $=\mathrm{F}$ is false in $\mathcal{M} 15$.

(iv) In the Second Fraenkel Model (Model $\mathcal{N} 2$ in [5]), MC is true but $\mathrm{DF}=\mathrm{F}$ is false (see [5]). It follows from part (ii) that "For every ultrafilter $\mathcal{U}$ on $\omega$ and every infinite set $A,\left|A^{\omega} / \mathcal{U}\right| \geq 2^{\aleph_{0}}$ " is also false in $\mathcal{N} 2$.

(v) From the proof of (i), the Basic Cohen Model witnesses the required independence result.

4. Open questions. Below, we list some open questions which naturally arise from the research in this paper.

Question 1. Does BPI imply "For every infinite set $X$, every almost disjoint family in $X$ can be extended to a MAD family in $X^{\prime \prime}$ ? Equivalently, does BPI imply "For every infinite set $X$, every antichain in the poset $\left(\mathcal{P}_{\infty}(X), \subseteq^{*}\right)$ can be extended to a maximal antichain"? (See Remark 3.)

QUESTION 2. Is there a model of ZFA or ZF in which "Every infinite set $X$ has an uncountable almost disjoint family" is true, but "Every infinite set $X$ has an uncountable $\left(>\aleph_{0}\right)$ almost disjoint family" is false? (Note that the second statement clearly implies the first one.)

Question 3. Does "Every infinite set $X$ has an uncountable $\left(>\aleph_{0}\right)$ almost disjoint family" imply "Every linearly ordered Dedekind-finite set is finite"?

QUESTION 4. What is the set-theoretic strength of the statement "Every infinite subset of $\mathbb{R}$ has an uncountable almost disjoint family"?

Acknowledgements. We are grateful to the anonymous referee for a careful review work; her/his apt comments and suggestions improved the quality and the exposition of our paper.

We would also like to express our gratitude to Professor Paul Howard for reading a former version of this paper, and especially for pointing out an error in the original proof of Theorem 6 and communicating a corrective argument, which we have incorporated with his kind permission. 


\section{References}

[1] N. Brunner, Dedekind-Endlichkeit und Wohlordenbarkeit, Monatsh. Math. 94 (1982), $9-31$.

[2] M. Droste and J. K. Truss, Subgroups of small index in ordered permutation groups, Quart. J. Math. Oxford Ser. (2) 42 (1991), 31-47.

[3] H. Höft and P. Howard, Well ordered subsets of linearly ordered sets, Notre Dame J. Formal Logic 35 (1994), 413-425.

[4] H. Horowitz and S. Shelah, On the non-existence of mad families, Arch. Math. Logic 58 (2019), 325-338.

[5] P. Howard and J. E. Rubin, Consequences of the Axiom of Choice, Math. Surveys Monogr. 59, Amer. Math. Soc., Providence, RI, 1998.

[6] T. J. Jech, The Axiom of Choice, Stud. Logic Found. Math. 75, North-Holland, Amsterdam, 1973.

[7] A. Lévy, Axioms of multiple choice, Fund. Math. 50 (1962), 475-483.

[8] A. R. D. Mathias, Happy families, Ann. Math. Logic 12 (1977), 59-111.

[9] D. Pincus, Adding dependent choice, Ann. Math. Logic 11 (1977), 105-145.

[10] E. Tachtsis, On the existence of free ultrafilters on $\omega$ and on Russell-sets in ZF, Bull. Polish Acad. Sci. Math. 63 (2015), 1-10.

[11] A. Törnquist, Definability and almost disjoint families, Adv. Math. 330 (2018), 61-73.

[12] J. K. Truss, Infinite permutation groups II. Subgroups of small index, J. Algebra 120 (1989), 494-515.

Eleftherios Tachtsis

Department of Statistics \& Actuarial-Financial Mathematics

University of the Aegean

Karlovassi 83200, Samos, Greece

E-mail: ltah@aegean.gr 